\title{
Combined Effect of Magnetic Field and Compressibility on Rayleigh Taylor Instability
}

\author{
Aniruddha Mitra1, Labakanta Mandal'2, Rajkumar Roychoudhury ${ }^{3}$, Manoranjan Khan' ${ }^{1}$ \\ ${ }^{1}$ Dept. of Instrumentation Science \& Centre for Plasma Studies, Jadavpur University, Kolkata, India \\ ${ }^{2}$ Dept. of Physics, Budge Budge Institute of Technology, Kolkata, India \\ ${ }^{3}$ Advanced Centre for Nonlinear \& Complex Phenomena, Kolkata, India \\ Email:anibabun@gmail.com, labakanta@gmail.com,rajdaju@rediffmail.com,mkhan.ju@yahoo.com
}

Received 13 October 2015; accepted 14 December 2015; published 18 December 2015

Copyright (C) 2015 by authors and Scientific Research Publishing Inc.

This work is licensed under the Creative Commons Attribution International License (CC BY). http://creativecommons.org/licenses/by/4.0/

(c) (i) Open Access

\begin{abstract}
The nonlinear analysis of the combined effect of magnetic field and compressibility on the growth rate of Rayleigh-Taylor (RT) instability has been investigated for inviscid two fluid interface. We have considered an interface-parallel density dependent magnetic field and used Layzer's approach to analyze the problem. We have also investigated the relative effect of magnetic pressure and hydrodynamic pressure on RT instability through the variation of the ratio of hydromagnetic pressure to magnetic pressure $(\beta)$. Dynamics of bubble and spike has been studied analytically and numerically. Finally, we have obtained the stability conditions of our result through linear stability analysis
\end{abstract}

\section{Keywords}

Rayleigh-Taylor Instability, Bubbles, Spikes, Self Generated Magnetic Field, Compressibility, Density Dependent Magnetic Field, ICF

\section{Introduction}

Under the gravitational force, when a denser fluid overlies a lighter fluid, the interfacial instability occurs between those two fluids, and it is called Rayleigh-Taylor instability (RTI). The development of nonlinear structures at the two fluid interface consequent to RTI is of much current interest both from theoretical and experimental points of view. The evolution of the structure is called a bubble if the lighter fluid pushes across the un- 
perturbed interface into the heavier fluid and it is termed as a spike if the opposite event takes place. The importance of such instabilities arises in connection with a wide range of applications ranging from astrophysical phenomena such as Supernova remnant to Inertial Confinement Fusion (ICF) problems. A core collapse Super Nova (SN) is driven by an external powerful shock, and strong shocks are the breeding ground of hydrodynamic instabilities like RTI. The interfacial RT instabilities have been investigated by several authors [1]-[11]. In an ICF situation, ablation front of an imploding capsule is subject to the RT instability because dense core is compressed and accelerated by low density ablating plasma. Recent experiments [12] [13] in LLNL shows that RTI at the ablation front of a composite ablator $(\mathrm{CH}, \mathrm{Si}$ dopped $\mathrm{CH})$ is induced, which requires mitigation for efficient ICF burn. Ponderomotive force due to spatially inhomogeneous laser beam is an effective mechanism for generation of large amplitude self generated magnetic field [14]-[16] in laser produced plasmas. Srivastava et al. [17] analyzed theoretically the spatially inhomogeneous laser beam could generate an axial magnetic field via ponderomotive force. The analytical results were compared with the experimental observation by [18]. The effect of self-generated magnetic field on RTI has been studied by Evans [19]. When the explosion of a Ia type supernova (SNIa) starts in a white dwarf as a laminar deflagration at the center of the star, RTI begins to act [20] [21]. In a white dwarf, magnetic field with strength up to $10^{8}-10^{9} \mathrm{G}$ exists at the surface and the field may be $\sim 10$ times greater near its center. RTI arising during type Ia Supernova explosion is also associated with strong magnetic field.

The effect of magnetic field on RTI was initially studied in details by [22]. In his linear analysis, he has shown that when the magnetic field is normal to the surface of separation of the two fluids, the RTI is shown to be unaffected by the magnetic field for small wave number " $k$ " of the perturbation. However, as $k$ increases, the RTI growth rate does not increase indefinitely with " $k$ " but tends to be a saturation value as $k \rightarrow \infty$ [22]. This situation may happen for sufficiently intense magnetic field.

If the external magnetic field is parallel to the interface, it is found in the linear theory that there exists no effect of the magnetic field on the instability [22]. However, in compressible and magnetized fluids, RTI has been shown to be reduced [23] even in linear theory. The RTI growth rate is found to be lowered for continuously accelerated two fluid interface when $k$ has component parallel to the magnetic field [24]. The nature of the depression has close resemblance to that of the effect of surface tension [22] and also has useful application in the astrophysical context [20] [25].

In all the above studies, the RTI was studied in linear approximation. However, in nonlinear theory, it has been studied separately, the evolution of the interfacial fluid structure due to RTI in compressible fluids [26] and that due to presence of magnetic field [27]. The present paper is addressed to the problem of the evolution of the nonlinear interfacial structure caused by RTI in presence of a magnetic field parallel to the surface of separation of the two compressible fluids. With such a geometry, there is no effect of the magnetic field in the classical linear approximation [22]. However, it is no longer the case for nonlinear theory. This may be understood from the following consideration.

In presence of only magnetic field [27], there exists a magnetic pressure in addition to the usual hydrodynamic pressure. As a result, the RTI driving pressure difference $g\left(\rho_{h}-\rho_{l}\right) y$ is changed by the inclusion of the magnetic pressure difference $(1 / 2 \mu)\left(B_{h}^{2}-B_{l}^{2}\right)$ [the suffix $\mathrm{h}(\mathrm{l})$ corresponds to the dynamical variable associated with the heavier (lighter) fluid]. In our earlier study [27] it has been shown that, the growth rate of RTI may be enhanced or depressed according to whether the extra contribution is positive or negative. Moreover, stabilization may occur in the sense that the surface of separation executes periodic undulation resulting from time lag in the temporal variation of $B_{h}$ and $B_{l}$. These are entirely nonlinear effects and disappear in the linear approximation.

In compressible fluids [26], there have been incorporated the effect of compressibility by introducing an extra work function proportional to surface pressure $p_{0}$ and density gradient scale length $\left(\delta \rho / \rho_{0}\right)$. The degree of stabilization or destabilization (depending on algebric sign of the density gradient at the surface) was shown to be proportional to $p_{0}$.

In this study, we have also included the compressibility in addition to the magnetic field on RTI. We have developed a model up to second order of nonlinearity in the transverse coordinate system to investigate the combined effect of compressibility and magnetic field by using Layzer's approach [28]. We have shown that, the growth rate of bubble and spikes is substantially reduced and shows oscillatory stabilization due to the combined effect which is conducive in ICF burn. We are mainly concentrated on a single mode bubble (spike) and our analysis is restricted in it (near the tip). The growth rate of instability is defined by the growth rate of the tip 
of the bubble (spike). The maximum spatial range of applicability of our solution has also been determined. We have tested the stability criteria of the solution.

The paper is organized as follows: Sec. 2 has been devoted for a brief discussion of Layzer's model. In Sec. 3 we have discussed the geometry of the problem and the structures of bubble and spike. Basic mathematical model and assumptions regarding the problem have been formulated in Sec. 4. The stability analysis of the model has been studied in Sec. 5. The numerical results regarding the dynamics of the tip of the bubble and spike have been discussed in Sec. 6. Sec. 7 is kept for conclusion.

\section{Layzer's Model: A Brief Discussion}

Potential flow model was first introduced in the nonlinear regime by Layzer in 1955 to study the stability of superposed fluid in a gravitation field in a three dimensional axially symmetric cylindrical or in two dimensional parallel walls from initially slight perturbed position. He obtained an approximate analytical solution considering a perfect incompressible fluid occupying the upper half of a vertical cylinder, being supported by a diaphragm against gravity. The lower half of the tube is empty i.e. $A_{T}=1$. The diaphragm is removed initially and applied a small disturbance at the interface then consequent motion has been investigated in 3D tubular and 2D periodic flows. He expanded the flow potentials using the Bessel (3D) and Fourier (2D) series, and retaining the lowest order terms, re-expanded the conservation laws in the vicinity of a regular point of the free surface (bubble tip). In this way, the nonlinear boundary value problem for the Euler equations was reduced to a system of ordinary differential equations.The asymptotic solutions for this dynamical system at short and long times have agreed with the linear theory of Rayleigh and with the results of Taylor, respectively. Though Layzers solution was the local one, as it described the temporal evolution of the flow in the vicinity of the bubble tip only, yet it accounted for the dynamics in the entire domain via the use of the Bessel and Fourier series .

\section{Geometrical Structure of the Problem}

We consider such a system in which heavier fluid lies above the lighter one and their interface is in the X-Z plane (Figure 1). Heavier fluid (density $\rho_{h}$ ) above this interface is extended to infinity towards positive $\mathrm{Y}$ axis and lighter fluid (density $\rho_{l}$ ) is below the interface and extended to infinity along negative Y axis. An external magnetic field has been applied across the interface towards positive $\mathrm{Z}$ axis. The gravity acts along negative $\mathrm{Y}$ axis. Since the force due to magnetic field is only in the perpendicular direction of the magnetic field, our study is essentially two dimensional.

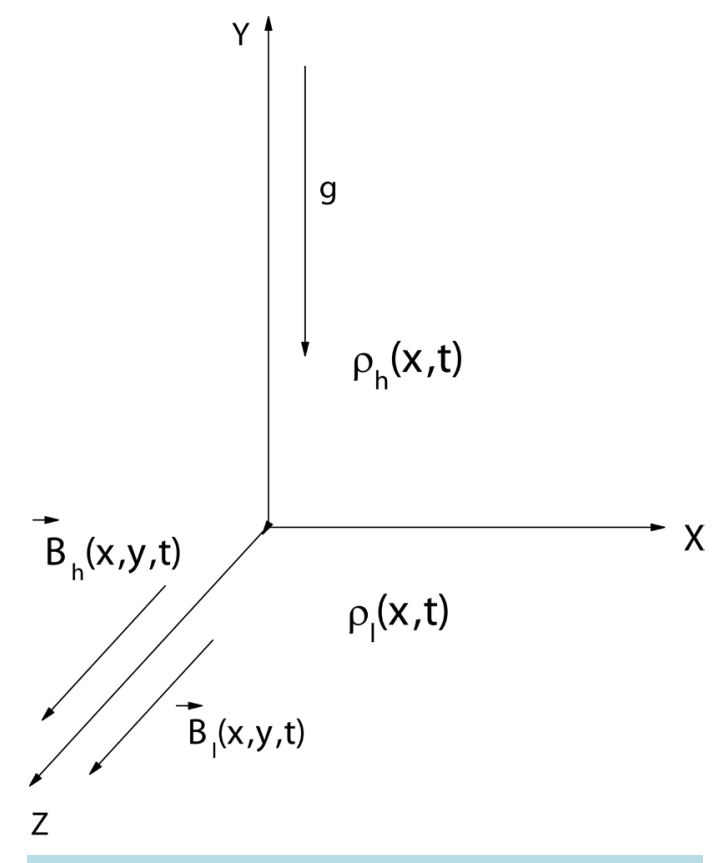

Figure 1. Geometry of the system. 
We assume the perturbed interface to be of parabolic form which is given by

$$
y(x, t)=\eta(x, t)=\eta_{0}(t)+\eta_{2}(t) x^{2}
$$

For a bubble (here the lower fluid is pushing across the interface into the upper fluid with $\rho_{h}>\rho_{l}$ ) we have,

$$
\eta_{0}>0 \text { and } \eta_{2}<0
$$

and for spike:

$$
\eta_{0}<0 \text { and } \eta_{2}>0
$$

here $\eta_{0}(t), \eta_{2}(t)$ are respectively, the height and curvature of the bubble.

\section{Basic Equations and Mathematical Model of Hydrodynamics}

We assume that the fluids are compressible, inviscid and the flow is irrotational. So there exists a velocity potential for both heavier and lighter fluids. Both the fluids obey the law of barotropic equation of state i.e. $p=c_{s}^{2} \rho^{\gamma}$, where $c_{s}$ is speed of sound through the fluid and $\gamma$ is adiabatic index of the fluid and $\rho$ is the density. The fluids are infinitely conducting in nature. We consider both high $\beta\left(p \gg B^{2} / 2 \mu\right)$ and low $\beta$ $\left(p \ll B^{2} / 2 \mu\right)$, where $\mathrm{p}$ is the fluid pressure and $\mathrm{B}$ is the magnetic field. We work in a frame in which the interface is at rest,so the zeroth order velocity $\boldsymbol{u}_{0}=0$ and we take the first order velocity to be $\boldsymbol{u}(x, y, t)$. We assume that the flow is irrotational. Moreover, in this situation the fluctuations are assumed to be incompressible, which may not require the fluid itself to be incompressible [29]. Thus we may consider the compressibility of the fluid in this condition. This assumption may be expressed as $\nabla \cdot \boldsymbol{u}=0$ i.e. $\nabla^{2} \phi=0$.

Following [1] velocity potential flow model, we get velocity potentials for heavier fluid as

$$
\phi_{h}(x, y, t)=a_{1}(t) \cos (k x) \mathrm{e}^{-k\left(y-\eta_{0}(t)\right)} ; y>0
$$

Velocity potential for lighter fluid is given by

$$
\phi_{l}(x, y, t)=b_{0}(t)+b_{1}(t) \cos (k x) \mathrm{e}^{k\left(y-\eta_{0}(t)\right)} ; y<0
$$

\subsection{Kinematic Boundary Condition}

In our study, we like to focus into the dynamics of the tip of the bubble and spike. Our whole analysis and results are concentrated on this particular dynamics of instability.

The kinematical boundary conditions satisfied at the interface $y=\eta(x, t)$ are

$$
\begin{gathered}
\frac{\partial \eta}{\partial t}-\frac{\partial \phi_{h}}{\partial x} \frac{\partial \eta}{\partial x}=-\frac{\partial \phi_{h}}{\partial y} \\
-\frac{\partial \phi_{h}}{\partial x} \frac{\partial \eta}{\partial x}+\frac{\partial \phi_{h}}{\partial y}=-\frac{\partial \phi_{1}}{\partial x} \frac{\partial \eta}{\partial x}+\frac{\partial \phi_{l}}{\partial y}
\end{gathered}
$$

Now substituting the values of $\phi_{h}, \phi_{1}$, and $\eta$ into above equations and expanding in powers of the transverse coordinate $\mathrm{x}$ and neglecting terms $O\left(x^{n}\right) \quad(n \geq 3)$ we get,

$$
\begin{gathered}
\frac{\mathrm{d} \xi_{1}}{\mathrm{~d} \tau}=\xi_{3} \\
\frac{\mathrm{d} \xi_{2}}{\mathrm{~d} \tau}=-3 \xi_{3}\left(\xi_{2}+\frac{1}{6}\right) \\
b_{0}=\left(\frac{-6 \xi_{2}}{3 \xi_{2}-0.5}\right) k a_{1}, \quad b_{1}=\left(\frac{3 \xi_{2}+0.5}{3 \xi_{2}-0.5}\right) a_{1}
\end{gathered}
$$

where $\xi_{1}=k \eta_{0} ; \xi_{2}=\eta_{2} / k ; \xi_{3}=k^{2} a_{1} / \sqrt{k g}$ are dimensionless displacement, curvature and velocity of the tip of 
the bubble, respectively.

\subsection{Governing Equations}

Basic equations to study our problem are : continuity equation, momentum equation, barotropic equation of state, and magnetic induction equation. The general forms of the equation are as follows:

$$
\begin{gathered}
\frac{\partial \rho}{\partial t}+\nabla \cdot(\rho \boldsymbol{V})=0 \\
\rho\left[\frac{\partial \boldsymbol{V}}{\partial t}+(\boldsymbol{V} \cdot \boldsymbol{\nabla}) \boldsymbol{V}\right]=-\nabla p-\rho \boldsymbol{g}+\frac{1}{\mu}(\nabla \times \boldsymbol{B}) \times \boldsymbol{B}
\end{gathered}
$$

Neglecting the magnetic tension term as the magnetic field to be planer, we get

$$
\rho\left[\frac{\partial \boldsymbol{V}}{\partial t}+(\boldsymbol{V} \cdot \nabla) \boldsymbol{V}\right]=-\nabla p-\rho \boldsymbol{g}-\nabla\left(\frac{B^{2}}{2 \mu}\right)
$$

and for infinitely conducting fluid

$$
\frac{\partial \boldsymbol{B}}{\partial t}=\nabla \times(\boldsymbol{V} \times \boldsymbol{B})
$$

Consequence of this infinite conductance is flux conservation (Alfven theorem), which tells that the the magnetic lines of force are frozen-in into the fluid.

\subsection{Time Evolution of Densities and Magnetic Fields}

Substituting the value of velocity potential for heavier fluid from Equation (4) into Equation (11), we have the following equation

$$
\frac{\partial \rho_{h}}{\partial t}-\nabla \phi_{h} \cdot \nabla \rho_{h}=0
$$

To solve this equation with the given form of velocity potential, we set,

$$
\rho_{h}(x, y, t)=\rho_{h 0}(t)+\left[\rho_{h 1}(t) \cos (k(x))\right] \mathrm{e}^{-k\left(y-\eta_{0}(t)\right)}
$$

Putting this form of density into Equation (15), and equating the coefficients of $x^{0}$ we get

$$
\rho_{h 0}(t)+\rho_{h 1}(t)=\tilde{\rho}_{h 0} \text { (constant) }
$$

Expanding Equation (16) up to $x^{2}$ order, we get the expression for density of the heavier fluid as follows

$$
\rho_{h}(x, y, t)=\tilde{\rho}_{h 0}-\rho_{h 1}(t) k^{2}\left(\xi_{2}+0.5\right) x^{2}
$$

where $\xi_{2}=\eta_{2} / k$.

Now equating the coefficients of $x^{2}$ in the Equation (15) we have,

$$
\frac{\dot{\rho}_{h 1}}{\rho_{h 1}}=\left[\frac{\xi_{2}-0.5}{\xi_{2}+0.5}\right] \xi_{3}
$$

where $\xi_{3}=a_{1} k^{2}$

In our model, we assume the magnetic field is not uniform throughout the system but depends on the density of the fluids. This is very much realistic because density dependent magnetic field may be present in Hybrid stars and Neutron stars [30]. To incorporate such a concept, we use the $\beta$ parameter ( ratio of hydrodynamic pressure and magnetic pressure) which allows us to extend our results from laboratory experiment to solar corona by just changing the value of $\beta$. So using Equation (17) and the expression for $\boldsymbol{B}$, viz $\boldsymbol{B}^{2}=\rho^{\gamma}\left[\left(2 \mu C_{s}^{2}\right) / \beta\right]$, we find 
the expression for magnetic field and its evolution for heavier density fluid as follows

$$
\boldsymbol{B}_{h}(t)=\tilde{\boldsymbol{B}}_{h 0}\left\{1-\frac{\gamma_{h} k^{2}}{2}\left(\frac{\boldsymbol{B}_{h 1}(t)}{\tilde{\boldsymbol{B}}_{h 0}}\right)^{\frac{2}{\gamma_{h}}}\left(\xi_{2}+0.5\right) x^{2}\right\}
$$

where

$$
\tilde{B}_{h 0}^{2}=\left(\frac{2 \mu C_{s h}^{2}}{\beta}\right)_{h} \tilde{\rho}_{h 0}^{\gamma_{h}}
$$

is magnetic field at equilibrium for heavier fluid. We also assume that the magnetic curvature force is too small in comparison with the magnetic pressure force. Time evolution of the magnetic field for heavier fluid is (using Equation (18)

$$
\frac{\dot{B}_{h 1}(t)}{B_{h}(t)}=\frac{\gamma_{h}}{2}\left(\frac{\xi_{2}-0.5}{\xi_{2}+0.5}\right) \xi_{3}
$$

Similarly for lighter fluid using Equation (5), Equation (15) and setting the density of lighter fluid as

$$
\rho_{l}(x, y, t)=\rho_{l 0}(t)+\rho_{l 1}(t) \cos (k x) \exp \left[k\left(y-\eta_{0}(t)\right)\right]
$$

we get the following form of density for lighter fluid (upto $x^{2}$ term)

$$
\rho_{l}(x, y, t)=\tilde{\rho}_{l 0}+\rho_{l 1}(t) k^{2}\left(\xi_{2}-0.5\right) x^{2}
$$

and the equation of time evolution of density is

$$
\frac{\dot{\rho}_{l 1}(t)}{\rho_{l 1}(t)}=\left(\frac{\xi_{2}+0.5}{\xi_{2}-0.5}\right)\left(\frac{\xi_{2}+\frac{1}{6}}{\xi_{2}-\frac{1}{6}}\right) \xi_{3}
$$

Similarly the value of magnetic field in lighter fluid is as follows

$$
\boldsymbol{B}_{l}(t)=\tilde{\boldsymbol{B}}_{l 0}\left\{1+\frac{\gamma_{l} k^{2}}{2}\left(\frac{B_{l 1}(t)}{\tilde{B}_{l 0}}\right)^{\frac{2}{\gamma_{l}}}\left(\xi_{2}-0.5\right) x^{2}\right\}
$$

and time evolution of this magnetic field is given by

$$
\frac{\dot{B}_{l}(t)}{B_{l}(t)}=\frac{\gamma}{2}\left(\frac{\xi_{2}+0.5}{\xi_{2}-0.5}\right)\left(\frac{\xi_{2}+\frac{1}{6}}{\xi_{2}-\frac{1}{6}}\right) \xi_{3}
$$

Now substituting $\boldsymbol{V}=-\nabla \phi$ into force equation Equation (13) and integrating once, we have,

$$
\frac{-\partial \phi}{\partial t}+\frac{1}{2}(\nabla \phi)^{2}=-\int \frac{\mathrm{d} p}{\rho}-\int \frac{\mathrm{d}\left(B^{2}\right)}{2 \mu \rho}+f(t)
$$

$f(t)$ is a time dependent integrating constant (independent of space variable). We denote the values of $f(t)$ as $f_{l}(t)$ and $f_{h}(t)$ for lighter and heavier fluid, respectively. However, $f_{l}(t)$ and $f_{h}(t)$ do not contribute anything in the dynamics of the interface both in linear and nonlinear theory [31]. Substituting the values of magnetic field, pressure, density for both fluids, we get two separate equations and subtracting them we get a 
combined equation as follows:

$$
\begin{aligned}
& \rho_{h 0}^{\star}\left[-\frac{\partial \phi_{h}}{\partial t}+\frac{1}{2}\left(\nabla \phi_{h}\right)^{2}+g y\right]-\rho_{l 0}^{\star}\left[-\frac{\partial \phi_{l}}{\partial t}+\frac{1}{2}\left(\nabla \phi_{l}\right)^{2}+g y\right] \\
& =-\left(p_{h 0}+\frac{\boldsymbol{B}_{h 0}^{2}}{\beta_{h}}\right)\left[1-\left(\gamma_{h}-1\right) \times \frac{\rho_{h 1}}{\tilde{\rho}_{h 0}} k^{2}\left(\xi_{2}+0.5\right) x^{2}\right] \\
& +\left(p_{l 0}+\frac{\boldsymbol{B}_{l 0}^{2}}{\beta_{l}}\right)\left[1+\left(\gamma_{l}-1\right) \frac{\rho_{l 1}}{\tilde{\rho}_{l 0}} k^{2}\left(\xi_{2}-0.5\right) x^{2}\right]+\rho_{h 0}^{\star} f_{h}(t)-\rho_{l 0}^{\star} f_{l}(t)
\end{aligned}
$$

where $\rho_{0}^{\star}=\tilde{\rho}_{0}(\gamma-1) / \gamma$. Now into the above equation, putting the values of

$$
\left(\nabla \phi_{h}\right)^{2},\left(\nabla \phi_{l}\right)^{2}, \partial \phi_{h} / \partial t, \partial \phi_{l} / \partial t
$$

and calculating only the coefficients of $x^{2}$ terms, we get

$$
\dot{\xi}_{3}=\chi_{1}+\chi_{2}+\chi_{3}
$$

where

$$
\begin{gathered}
\chi_{1}=-\frac{N\left(\xi_{2}, r^{*}\right)}{D\left(\xi_{2}, r^{*}\right)} \frac{\xi_{3}^{2}}{6 \xi_{2}-1} \\
\chi_{2}=2 k g\left(r^{*}-1\right) \frac{\xi_{2}\left(6 \xi_{2}-1\right)}{D\left(\xi_{2}, r^{*}\right)} \\
\chi_{3}=-\frac{6 \xi_{2}-1}{D\left(\xi_{2}, r^{*}\right)} \pi_{1}-\frac{6 \xi_{2}-1}{D\left(\xi_{2}, r^{*}\right)} \pi_{2} \\
\pi_{1}=\left(\frac{r^{*} \gamma_{h} k^{2}}{\tilde{\rho}_{h 0}}\left(p_{h 0} \delta \rho_{h}(t)+\frac{B_{h 0}^{2}}{\beta_{h}}\left(\delta B_{h}(t)\right)^{\frac{2}{\gamma_{h}}}\right)\left(\xi_{2}+0.5\right)\right) \\
\pi_{2}=\left(\frac{\gamma_{l} k^{2}}{\tilde{\rho}_{l 0}}\left(p_{l 0} \delta \rho_{l}(t)+\frac{\boldsymbol{B}_{l 0}^{2}}{\beta_{l}}\left(\delta B_{l}(t)\right)^{\frac{2}{\gamma_{l}}}\right)\left(\xi_{2}-0.5\right)\right) \\
\frac{\rho_{h 1}(t)}{\rho_{h 0}}=\delta \rho_{h}(t) ; \frac{\rho_{l 1}(t)}{\rho_{l 0}}=\delta \rho_{l}(t) ; \frac{B_{h 1}(t)}{B_{h 0}}=\delta B_{h}(t) ; \\
\frac{B_{l 1}(t)}{B_{l 0}}=\delta B_{l}(t) ; r^{*}=\frac{\rho_{h 0}^{\star}}{\rho_{l 0}^{\star}} \\
N\left(\xi_{2}, r^{*}\right)=36\left(1-r^{*}\right) \xi_{2}^{2}+12\left(4+r^{*}\right) \xi_{2}+\left(7-r^{*}\right) \\
D\left(\xi_{2}, r^{*}\right)=12\left(1-r^{*}\right) \xi_{2}^{2}+4\left(1-r^{*}\right) \xi_{2}+\left(1+r^{*}\right)
\end{gathered}
$$

Finally we get the full set of equations as follows

$$
\begin{gathered}
\dot{\xi}_{1}=\xi_{3} \\
\dot{\xi}_{2}=-\frac{1}{2}\left(6 \xi_{2}+1\right) \xi_{3} \\
\dot{\xi}_{3}=\chi_{1}+\chi_{2}+\chi_{3}
\end{gathered}
$$




$$
\begin{gathered}
\frac{\dot{\rho}_{l 1}(t)}{\rho_{l 1}(t)}=\left(\frac{\xi_{2}+0.5}{\xi_{2}-0.5}\right)\left(\frac{\xi_{2}+\frac{1}{6}}{\xi_{2}-\frac{1}{6}}\right) \xi_{3} \\
\frac{\dot{\rho}_{h 1}}{\rho_{h 1}}=\left(\frac{\xi_{2}-0.5}{\xi_{2}+0.5}\right) \xi_{3} \\
\frac{\dot{B}_{h 1}(t)}{B_{h}(t)}=\frac{\gamma_{h}}{2}\left(\frac{\xi_{2}-0.5}{\xi_{2}+0.5}\right) \xi_{3} \\
\frac{\dot{B}_{l 1}(t)}{B_{l}(t)}=\frac{\gamma}{2}\left(\frac{\xi_{2}+0.5}{\xi_{2}-0.5}\right)\left(\frac{\xi_{2}+\frac{1}{6}}{\xi_{2}-\frac{1}{6}}\right) \xi_{3}
\end{gathered}
$$

The above set of equations (26) describe the time evolution of bubble.

The time evolution equation for spike can be obtained by making the following transformations [7]: $\xi_{1} \rightarrow-\xi_{1}, \quad \xi_{2} \rightarrow-\xi_{2}, \quad \xi_{3} \rightarrow \xi_{3}$ and $r^{*} \rightarrow 1 / r^{*}$.

$$
\begin{gathered}
\dot{\xi}_{1}=-\xi_{3} \\
\dot{\xi}_{2}=\frac{1}{2}\left(1-6 \xi_{2}\right) \xi_{3} \\
\dot{\xi}_{3}=\tilde{\chi}_{1}+\tilde{\chi}_{2}+\tilde{\chi}_{3} \\
\frac{\dot{\rho}_{l 1}(t)}{\rho_{l 1}(t)}=\left(\frac{\xi_{2}-0.5}{\xi_{2}+0.5}\right)\left(\frac{\xi_{2}-\frac{1}{6}}{\xi_{2}+\frac{1}{6}}\right) \xi_{3} \\
\frac{\dot{\rho}_{h 1}}{\rho_{h 1}}=\left(\frac{\xi_{2}+0.5}{\xi_{2}-0.5}\right) \xi_{3} \\
\frac{\dot{B}_{h 1}(t)}{B_{h}(t)}=\frac{\gamma_{h}}{2}\left(\frac{\xi_{2}+0.5}{\xi_{2}-0.5}\right) \xi_{3} \\
\frac{\dot{B}_{l 1}(t)}{B_{l}(t)}=\frac{\gamma}{2}\left(\frac{\xi_{2}-0.5}{\xi_{2}+0.5}\right)\left(\frac{\xi_{2}-\frac{1}{6}}{\xi_{2}+\frac{1}{6}}\right) \xi_{3}
\end{gathered}
$$

where

$$
\begin{gathered}
\chi_{1}=\frac{N\left(\xi_{2}, 1 / r^{*}\right)}{D\left(\xi_{2}, 1 / r^{*}\right)} \frac{\xi_{3}^{2}}{6 \xi_{2}+1} \\
\chi_{2}=2 k g\left(1-1 / r^{*}\right) \frac{\xi_{2}\left(6 \xi_{2}+1\right)}{D\left(\xi_{2}, 1 / r^{*}\right)} \\
\chi_{3}=\frac{6 \xi_{2}+1}{D\left(\xi_{2}, 1 / r^{*}\right)} \tilde{\pi}_{1}+\frac{6 \xi_{2}+1}{D\left(\xi_{2}, 1 / r^{*}\right)} \tilde{\pi}_{2}
\end{gathered}
$$




$$
\begin{gathered}
\tilde{\pi}_{1}=-\frac{\gamma_{h^{*}} k^{2}}{r^{*} \tilde{\rho}_{h 0}}\left(p_{h 0} \delta \rho_{h}(t)+\frac{\boldsymbol{B}_{h 0}^{2}}{\beta_{h}}\left(\delta B_{h}(t)\right)^{\frac{2}{\gamma_{h}}}\right)\left(\xi_{2}-0.5\right) \\
\tilde{\pi}_{2}=-\frac{\gamma_{l} k^{2}}{\tilde{\rho}_{l 0}}\left(p_{l 0} \delta \rho_{l}(t)+\frac{\boldsymbol{B}_{l 0}^{2}}{\beta_{l}}\left(\delta B_{l}(t)\right)^{\frac{2}{\gamma_{l}}}\right)\left(\xi_{2}+0.5\right) \\
N\left(\xi_{2}, 1 / r^{*}\right)=36\left(1-1 / r^{*}\right) \xi_{2}^{2}+12\left(4+1 / r^{*}\right) \xi_{2}+\left(7-1 / r^{*}\right) \\
D\left(\xi_{2}, 1 / r^{*}\right)=12\left(1-1 / r^{*}\right) \xi_{2}^{2}+4\left(1-1 / r^{*}\right) \xi_{2}+\left(1+1 / r^{*}\right)
\end{gathered}
$$

\section{Stability Analysis}

We check the stability of the system given by the set of equations Equation (26), assuming the initial values $\rho_{h 0} / \tilde{\rho}_{h 0}$ and $\rho_{l 0} / \tilde{\rho}_{l 0}$ to be unity. Let us consider the following coupled equations,

$$
\dot{\xi}_{3}=\tilde{\chi}_{1}+\tilde{\chi}_{2}+\tilde{\chi}_{3}
$$

and

$$
\dot{\xi}_{2}=-\frac{1}{2}\left(6 \xi_{2}+1\right) \xi_{3}=g\left(\xi_{2}, \xi_{3}\right)(\text { say })
$$

There are two sets of fixed points of the system given by the above equations, are

$$
\left\{\xi_{2}=\frac{1}{6}, \xi_{3}=0\right\}
$$

and

$$
\left\{\xi_{2}=\frac{1}{2}\left[\frac{r^{*} \alpha_{h}-\alpha_{l}}{\frac{2 g}{k}\left(r^{*}-1\right)-r \alpha_{h}-\alpha_{l}}\right], \xi_{3}=0\right\}
$$

where

$$
\begin{gathered}
\alpha_{h}=C_{s h}^{2}+\frac{\gamma_{h} \mu_{h}}{\beta_{h}} V_{a h}^{2} ; \\
\alpha_{l}=C_{s l}^{2}+\frac{\gamma_{l} \mu_{l}}{\beta_{l}} V_{a l}^{2} \\
C_{s}=\sqrt{\frac{\gamma p}{\rho}} \text { (sound velocity) } \\
V_{a}=\sqrt{\frac{B^{2}}{\mu \rho}} \text { (Alfven velocity) }
\end{gathered}
$$

Now to check the stability of the solutions we calculate the eigen values of the following matrix, called Jacobian $(J)$, at those fixed points

$$
J=\left(\begin{array}{ll}
\frac{\partial g}{\partial \xi_{2}} & \frac{\partial g}{\partial \xi_{3}} \\
\frac{\partial h}{\partial \xi_{2}} & \frac{\partial h}{\partial \xi_{3}}
\end{array}\right)
$$


If $\lambda$ be the eigen value of the Jacobian, then

$$
\left(\begin{array}{cc}
\frac{\partial g}{\partial \xi_{2}}-\lambda & \frac{\partial g}{\partial \xi_{3}} \\
\frac{\partial h}{\partial \xi_{2}} & \frac{\partial h}{\partial \xi_{3}}-\lambda
\end{array}\right)=0
$$

The eigen values at the fixed point $\xi_{2}=1 / 6, \xi_{3}=0$ are

$$
\left\{\frac{1}{2} \varpi,-\frac{1}{2} \varpi\right\}
$$

where

$$
\varpi=\sqrt{6\left[r^{*} \alpha_{h}+\alpha_{l}-k g\left(r^{*}-1\right)\right]}
$$

So, the system will be stable if

$$
r^{*} \alpha_{h}<k g\left(r^{*}-1\right)-\alpha_{l}
$$

The above mentioned condition also holds for the stability of the fixed point

$$
\left(\xi_{2}=\frac{1}{2}\left\{\frac{r^{*} \alpha_{h}-\alpha_{1}}{\frac{2 g}{k}\left(r^{*}-1\right)-r^{*} \alpha_{h}-\alpha_{l}}\right\}, \xi_{3}=0\right)
$$

This has been checked thoroughly by using numerical method. The phase diagram (Figure 2) also ensures the stability of the system under that above mentioned condition.

However, the eigen value of the second set of fixed point is of very lengthy expression and not presented in this paper.

\section{Numerical Results and Discussion}

According to the linear analysis,when the externally applied magnetic field is parallel to the interface of separation of the two fluid system (heavier fluid lies over the lighter fluid under the action of gravity), the RTI remain unaffected [22]. However, nonlinear analysis shows a decreasing nature of RTI growth rate for the above configuration [27]. It was shown that, compressibility also decreases the growth rate of RTI [26] [32]. In our

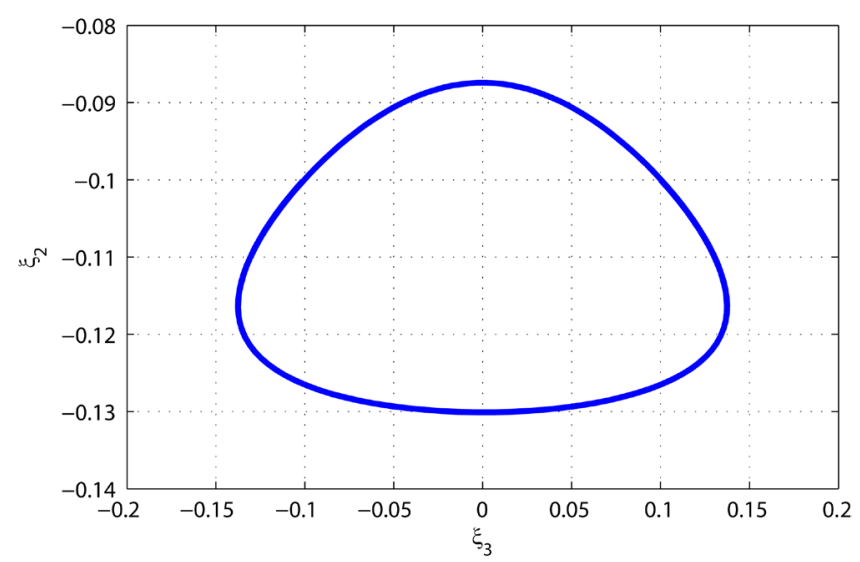

Figure 2. Phase diagram with initial values $\xi_{1}=-\xi_{2}=\xi_{3}=-0.1$

and $r=1.5$. 
nonlinear study, we consider the compressibility and interface-parallel magnetic field simultaneously, which has not been studied previously.

To verify our model numerically, we have used Runge-Kutta method and set the values of constants in our equations as follows: $k g=1, \beta_{h}=\beta_{l}$,

The results of our studies are discussed as follows.

\subsection{Bubble}

1) From Figure 3, it is clear that, both compressibility and magnetic field decreases the RTI growth rate separately. When they act together the growth rate decreases and system shows oscillatory stabilization. The reason behind such oscillatory stabilization is as follows : When both fluids are conducting, the hydrodynamic pressure difference and magnetic pressure difference for both fluids acts in opposite direction on two sides of the interface. The resultant force due to those two pressure differences induces an oscillatory motion at the interface. The amplitude and the velocity of that oscillatory motion are finite but change rapidly in periodic manner. Moreover, the magnetic pressure difference brings all the periodicity in the motion. The force due to hydrodynamic pressure difference can not lead the system to the oscillatory stabilization, alone.

2) Figure 4 shows the relative effect of magnetic field strength and compressibility on the growth rate of RTI. The advantage of our model is that, we can observe the domination of magnetic field or hydrodynamic pressure force on the growth rate of instability through the changing value of $\beta$. When $\beta$ is small, the magnetic field dominates the dynamics and when $\beta$ is large $(>1)$ the hydrodynamic pressure dominates the dynamics of instability. As we mentioned earlier the dynamics of the interface is governed by the resultant force due to compressibility (hydrodynamic force) and magnetic pressure (magnetic force). We can see from Figure 4 that, as $\beta$ increases, the oscillatory nature of the motion of the interface decreases. The reason behind this changes is as follows: when $\beta$ increases the effect of magnetic field decreases and hydrodynamic pressure force increases. As we stated earlier, the periodicity of the motion occurs due to the magnetic field solely. So, as $\beta$ increases i.e. the magnetic force decreases the periodicity of the motion decreases and the motion becomes hyd-
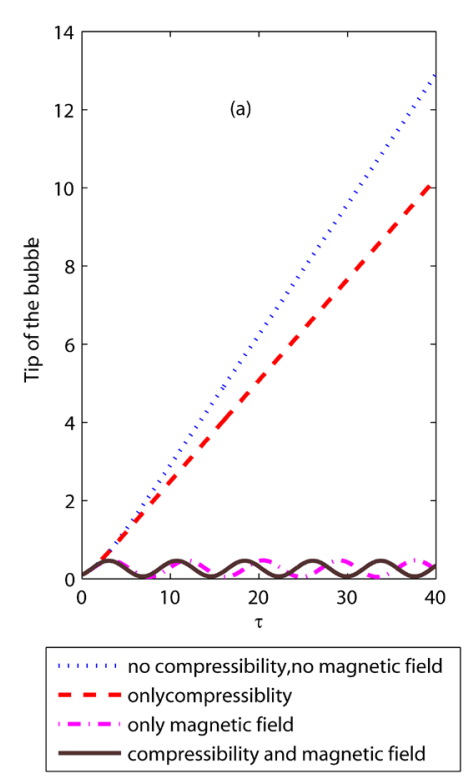
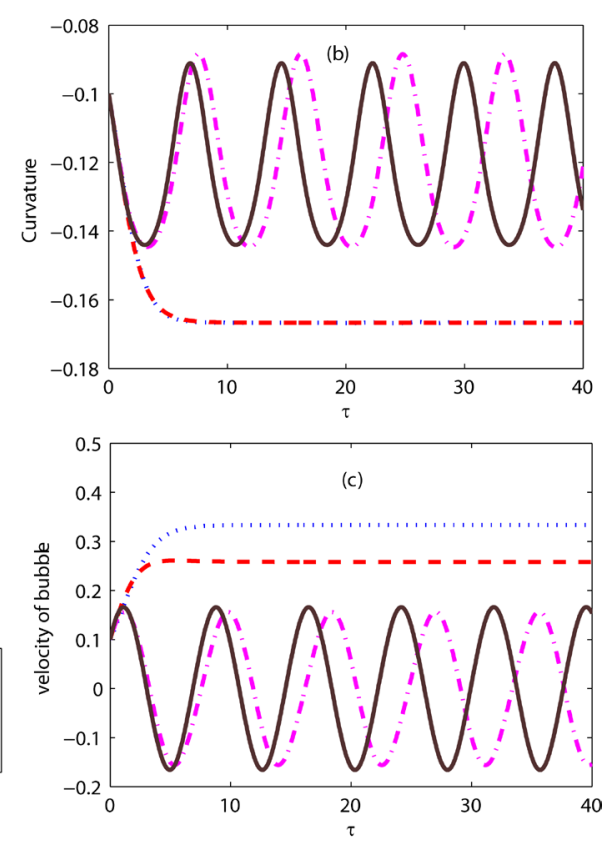

Figure 3. Variation of (a) height of tip $\left(\xi_{1}\right)$, (b) curvature $\left(\xi_{2}\right)$ and (c) velocity $\left(\xi_{3}\left(=\dot{\xi}_{1}\right)\right)$ of bubble in presence of compressibility and magnetic field. Initial values $\xi_{1}=-\xi_{2}=\xi_{3}=-0.1$ and $r=1.5, \beta_{h}=\beta_{l}=1.0, \gamma_{h}=\gamma_{l}=1.0$ (for incompressible case) and $\gamma_{h}=\frac{5}{3} ; \gamma_{l}=\frac{3}{2}$ (for compressible case), $A_{T}=0.20$. 

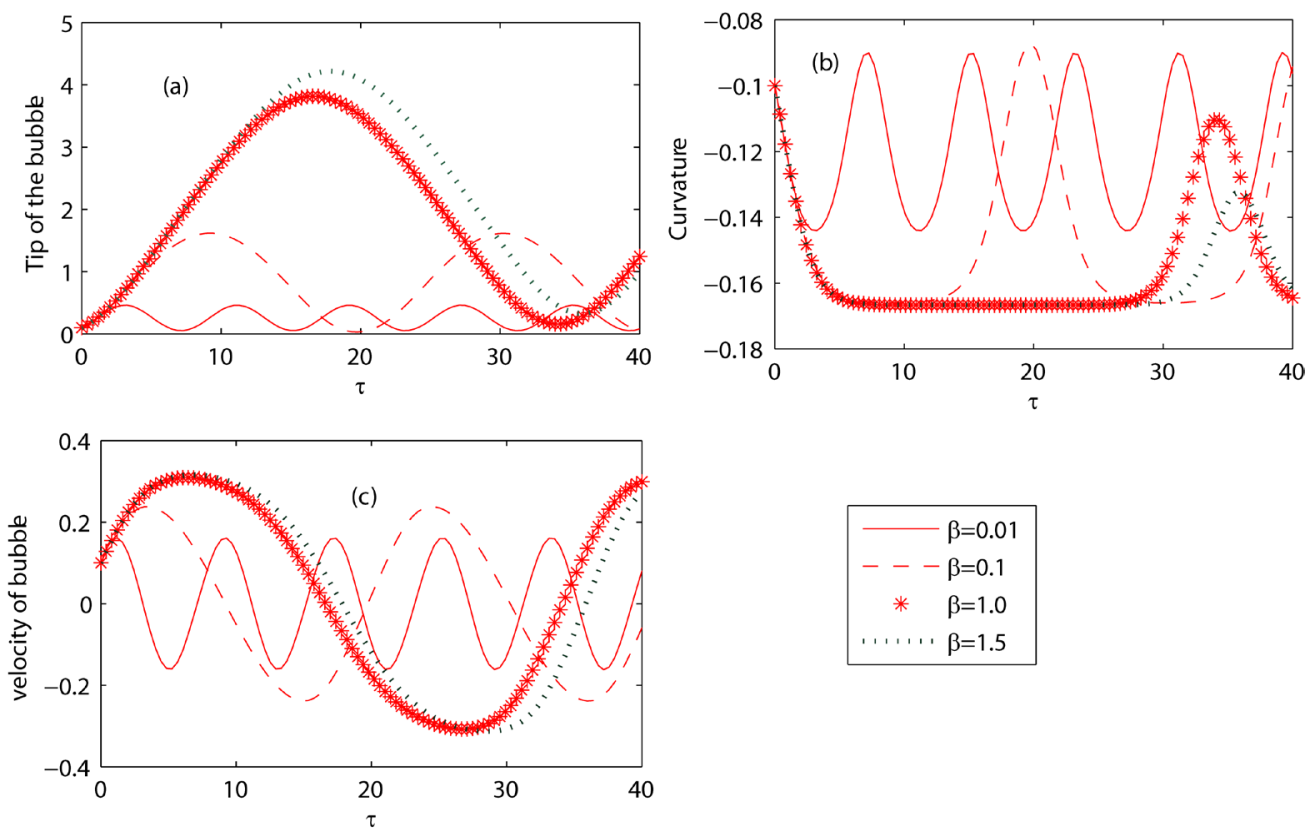

Figure 4. Variation of (a) height of tip $\left(\xi_{1}\right)$, (b) curvature $\left(\xi_{2}\right)$ and (c) velocity $\left(\xi_{3}\left(=\dot{\xi}_{1}\right)\right)$ of
bubble in presence of compressibility and magnetic field for different values of $\beta\left(\beta_{h}=\beta_{l}\right)$. Initial
values $\xi_{1}=-\xi_{2}=\xi_{3}=-0.1$ and $r=1.5, A_{T}=0.20$.

rodynamic force dominant. According to our analysis, for a fixed value of Atwood number $\left(A_{T}\right)$, their is a certain critical value of $\beta\left(=\beta_{c}\right)$, above which the motion of the interface loses its periodic nature and become completely unstable. We show this for a critical beta value $\beta_{c}=500$ at $A_{T}=0.20$ (Figure 5).

3) The effect of variation of Atwood number on the dynamics of instability has been shown in the Figure 6. For a fixed value of $\beta$, the growth rate of instability increases with increasing value of Atwood number. As energy is conserved in the system, the height of the bubble decreases as velocity increases with increasing value of Atwood number. This result is obvious because, as Atwood number increases, the density difference increases, which ensure a lower buoyancy force on the upper (heavier) fluid due to the lower (lighter) fluid. A lower buoyancy force means a lower resistance for the penetration of heavier fluid into the lighter one. As the total volume of the system is conserved, the lighter fluid penetrates into the heavier fluid in the form of bubble. So, as Atwood number increases, the buoyancy force decreases, the growth rate of bubble increases; our numerical analysis (Figure 6) ensures this conclusion.

\subsection{Spike}

When heavier fluid penetrates into the lighter fluid under the action of some acceleration (here gravity), spikes form. We will discuss here the results of our analysis about spike.

1) From Figure 7 it is clear that, under the action of an oscillatory magnetic field, the height, curvature and velocity of the spike changes in periodic manner with finite amplitude. At the first stage, when magnetic pressure increases, the height increases but curvature decreases almost exponentially. The reason behind this is the following. As the magnetic field is parallel to the interface and depends on the densities of the fluids, total pressure on the interface increases in the opposite direction of density gradient. As a result, the heavier fluid falls rapidly into the lighter fluid with large amplitude in the form of spike. The decreasing character of the curvature of the spike implies that, the spike become narrower. This increasing height and decreasing curvature is the consequence of conservation of total volume of the system. In the next stage, where the magnetic field is almost constant, the height, curvature and velocity also remain unchanged. At the last stage of a complete magnetic cycle, the height decreases and curvature increases. This is again due to the conservation of total volume of the system. However, at this stage, the velocity of the spike changes significantly. For first few moments of this 

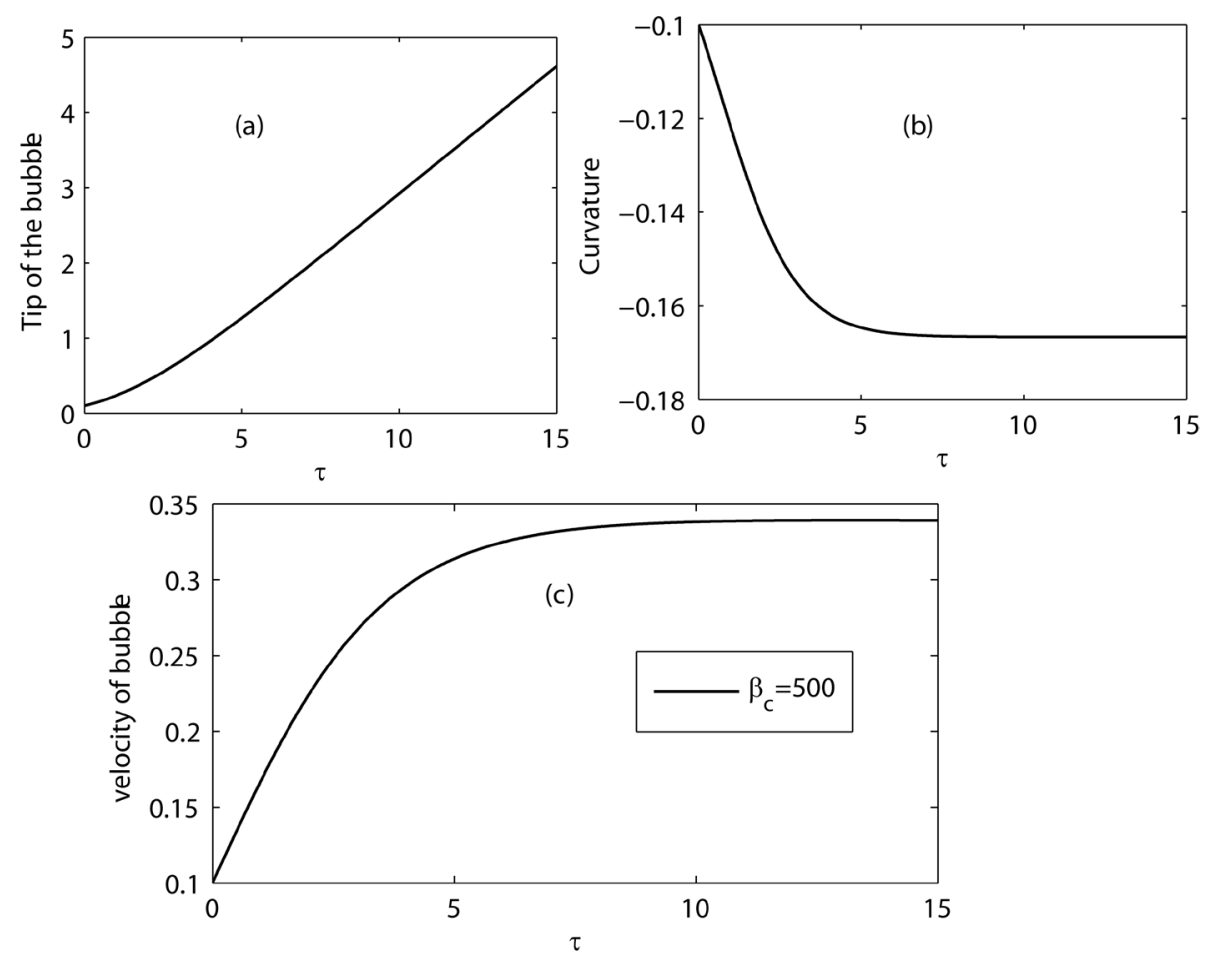

Figure 5. Variation of (a) height of tip $\left(\xi_{1}\right)$, (b) curvature $\left(\xi_{2}\right)$ and (c) velocity $\left(\xi_{3}\left(=\dot{\xi}_{1}\right)\right)$ of bubble at $\beta\left(\beta_{h}=\beta_{l}=\beta_{h c}=500\right)$. Initial values $\xi_{1}=-\xi_{2}=\xi_{3}=-0.1$ and $A_{T}=0.20, r=1.5$.
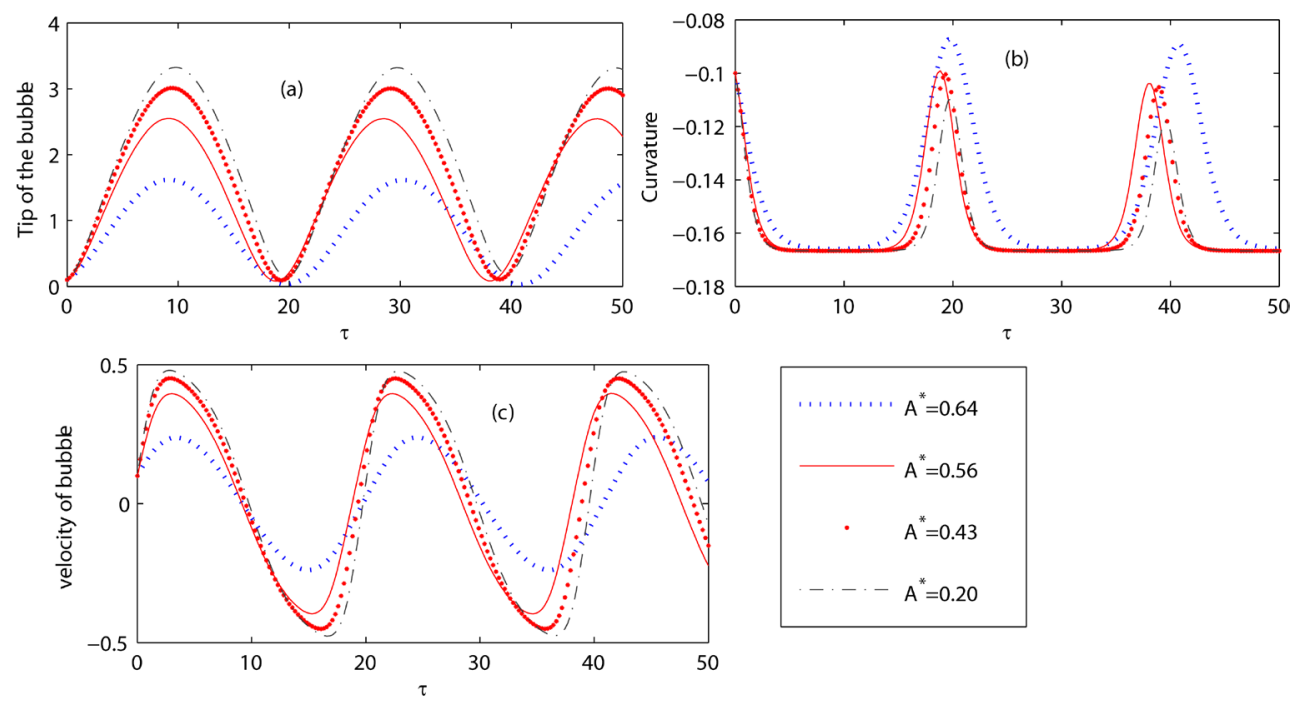

Figure 6. Variation of (a) height of tip $\left(\xi_{1}\right)$, (b) curvature $\left(\xi_{2}\right)$ and (c) velocity $\left(\xi_{3}\left(=\dot{\xi}_{1}\right)\right)$ of bubble for different values of Atwood number. Initial values $\xi_{1}=-\xi_{2}=\xi_{3}=-0.1$.

phase, velocity increases almost linearly. We believe that this sudden rise of velocity is due to a rapid compression of density of the fluids. The final stage of this phase is characterized by rapid expansion of density and subsequent fall of spike velocity.

This whole cycle of changes in height, curvature and velocity of the spike, repeats itself throughout the evolution of instability. Moreover we can see from the same figure (Figure 7) that, as magnetic field decreases 

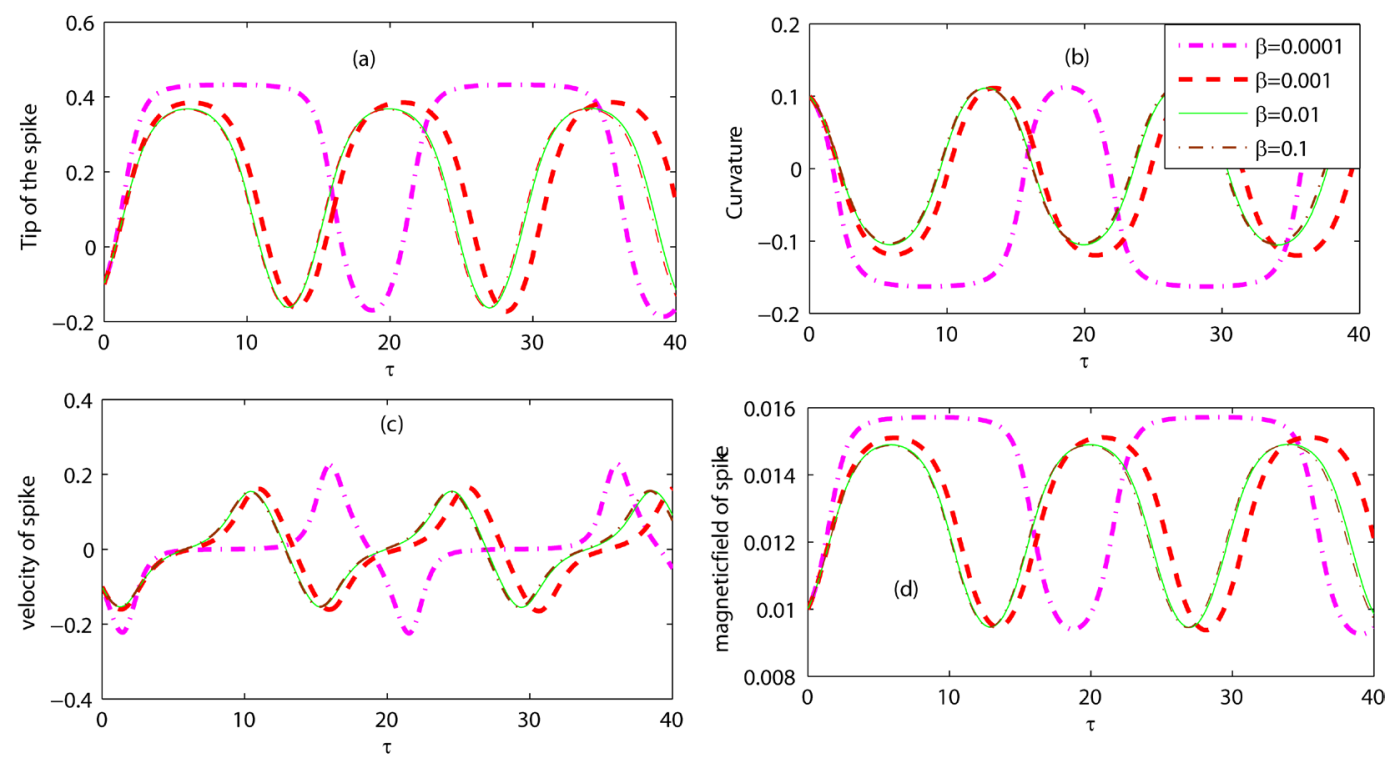

Figure 7. Variation of (a) height of tip $\left(\xi_{1}\right)$, (b) curvature $\left(\xi_{2}\right)$ and (c) velocity $\left(\xi_{3}\left(=\dot{\xi}_{1}\right)\right)$ and (d) magnetic field of spike with the value of different $\beta\left(\beta_{h}=\beta_{l}\right)$. Initial values $-\xi_{1}=-\xi_{3}=-0.1 ;-\xi_{2}=0.1$ and $r=\frac{1}{1.5}, A_{T}=0.20, \gamma_{h}=\frac{5}{3} ; \gamma_{l}=\frac{3}{2}$.

i.e. $\beta$ increases, the stable region of spike velocity decreases and height of the spike decreases.

2) Figure 8 shows the dependence of the height, curvature and velocity of spike on Atwood number $\left(A_{T}\right)$. The velocity of the spike decreases as $A_{T}$ decreases in oscillatory manner. For a fixed value of $A_{T}$, velocity decreases however, height and curvature of the spike increases for a short period of time. After that, velocity increases very slowly but height of the spike remain almost unchanged over a long time period. After this long time range, the velocity and height of the spike decreases very rapidly over a small time scale. The highest value of spike velocity depends on $A_{T}$. The maximum attainable value of spike velocity increases with increasing $A_{T}$. This is very natural because, the hydrodynamic pressure on the interface increases with increasing value of $A_{T}$, so spike falls more rapidly against gravity.

3) In case of spike, the critical value of magnetic field (at which the spike become unstable) depends largely on Atwood number. As we can see from Figure 9 that, $\beta=0.0001$ becomes the critical value for all Atwood number $\left(A_{T}\right)$ greater than 0.5, however, $\beta=0.0001$ is not the critical value for $A_{T}=0.20$.

\section{Conclusions}

We found in earlier studies that interface parallel magnetic field decreases the growth rate nonlinearly, whereas, compressibility effect also linearly decreases the RTI growth rate for bubble and spikes. When they act together, the resultant decreasing character of growth rate depends on their relative strength. This relative strength depends on the value of $\beta$. If $\beta$ is small, i.e. $\beta<1$, the magnetic field dominates the growth rate when $\beta>1$ compressibility has the dominating effect on the growth rate.

In our calculation, we assume the variation of $\beta$ for both fluids are negligible and their values are almost constant. We also see that, Atwood number $\left(A_{T}\right)$ has very profound effect on the dynamics of instability. For fixed value of magnetic field, $A_{T}$ governs the dynamics of the interface. Moreover, in presence of magnetic field, the evolution of the bubble is quite different from that of the spike, near the critical $\beta$ value, for a fixed $A_{T}$.

In this paper we have considered single mode bubble (spike) and the whole analysis is valid for the spatial scale lengths $0<x \leq \frac{1}{k} \sqrt{\frac{2 \tilde{\rho}_{h(l) 0}}{\rho_{h(l) 1}(t)\left(2 \xi_{2}+1\right)}}$. This particular limit in length scale arises due to our assumption 

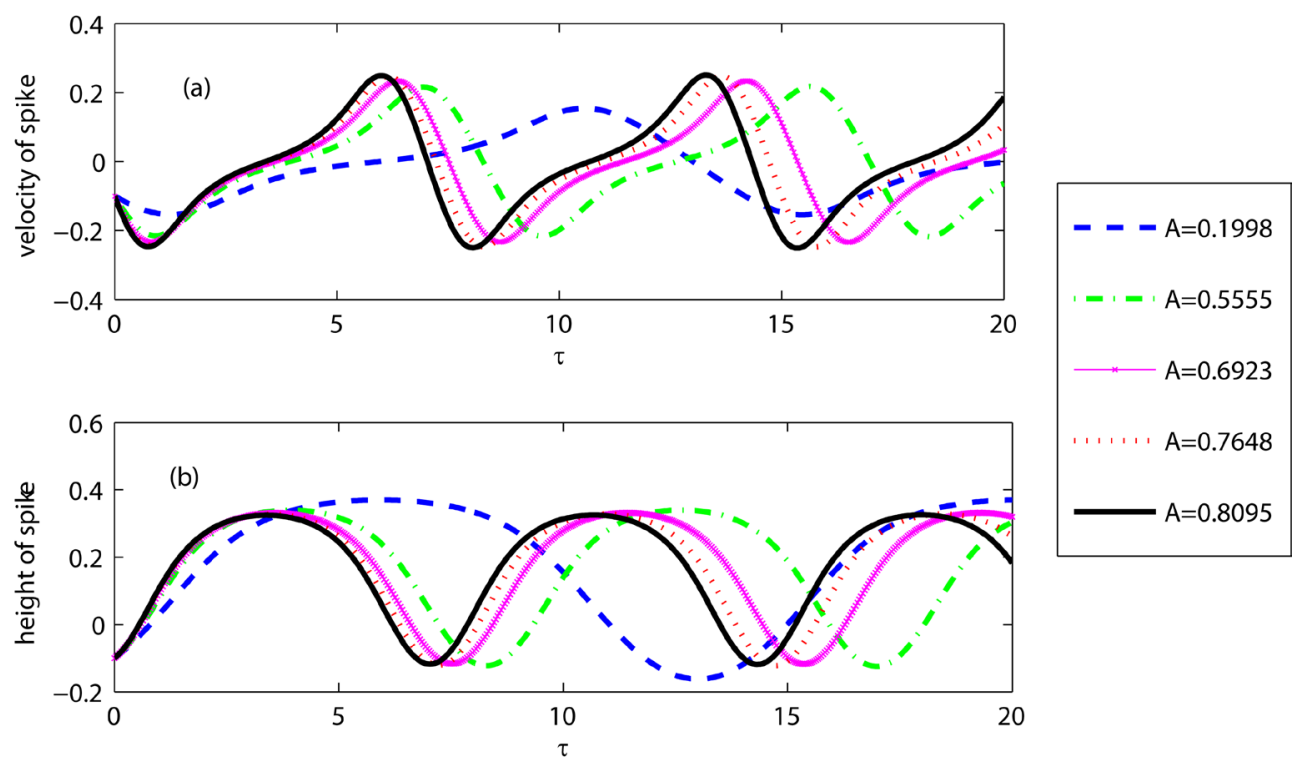

Figure 8. Ariation of (a) velocity $\left(\xi_{3}\left(=\dot{\xi}_{1}\right)\right)$ and (b) height of tip $\left(\xi_{1}\right)$ of spike for different value of Atwood number (A). Initial values $-\xi_{1}=-\xi_{3}=-0.1 ;-\xi_{2}=0.1$ and $r=\frac{1}{1.5}, \quad \beta_{h}=\beta_{l}=1.0$, $\gamma_{h}=\frac{5}{3} ; \gamma_{l}=\frac{3}{2}$.
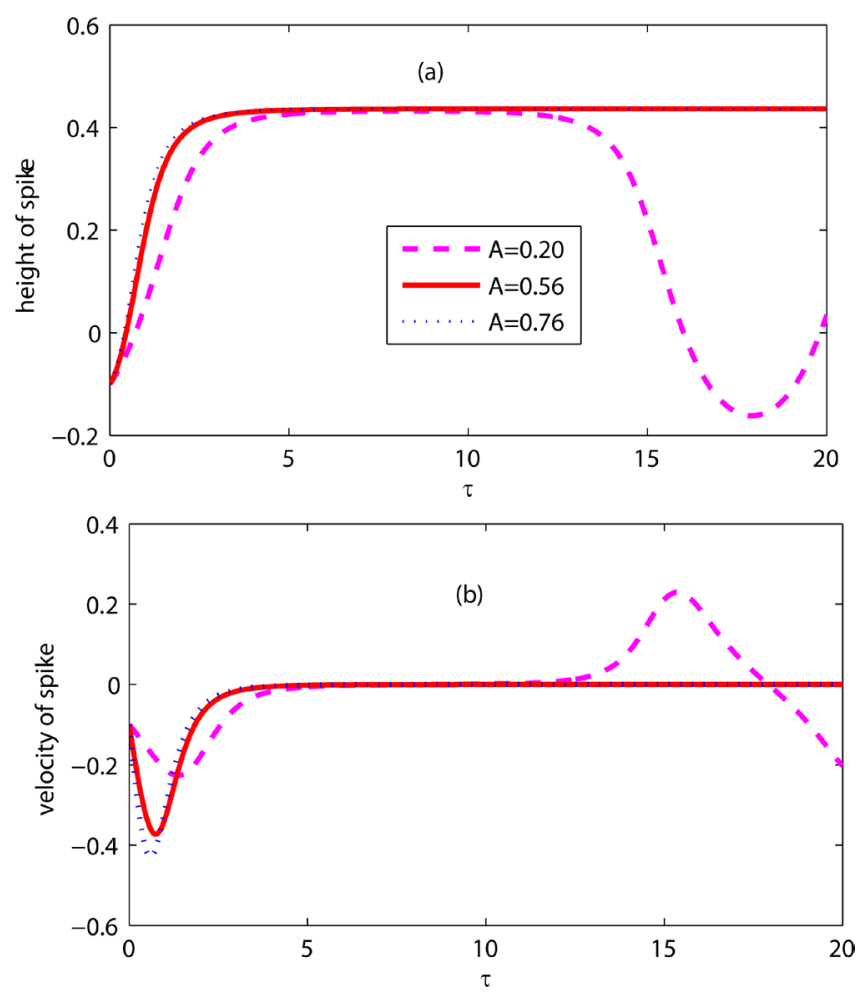

Figure 9. Variation of (a) height of tip $\left(\xi_{1}\right)$ and (b) velocity $\left(\xi_{3}\left(=\dot{\xi}_{1}\right)\right)$ of at a fixed value of $\beta\left(\beta_{h}=\beta_{1}=0.0001\right)$. Initial $-\xi_{1}=-\xi_{3}=-0.1 ;-\xi_{2}=0.1, \gamma_{h}=\frac{5}{3} ; \gamma_{1}=\frac{3}{2}, \quad r=\frac{1}{1.5}$. 
about the shape of the bubble and spike as a parabola and also the fact that we ignored the higher order terms in the expansion of velocity potential. However, this length scale gives an overview of the effect of magnetic field and compressibility on the growth rate of RT instability. From our general model of RT instability, all previous results concerning effect of a) only compressibility [26] [32]; b) only magnetic field [27] can be derived, shown in the numerical graphs (Figure 3, Figure 4, Figures 6-8).

Recent experiments [12] [13] on hydrodynamic instability and mix experiments at Nation Ignition Facilities (NIF) reveal that such RTI at the ablation front in composite ablator is primarily responsible for the hot spot mix, which requires mitigation. In our analysis, it is evident that the compressibility in the composite ablator and magnetic field may play a key role in mitigating RTI leading to ignition at the hot spot.

\section{References}

[1] Goncharov, V.N. (2002) Analytical Model of Nonlinear, Single-Mode, Classical Rayleigh-Taylor Instability at Arbitrary Atwood Numbers. Physical Review Letters, 88, Article ID: 134502.

http://dx.doi.org/10.1103/PhysRevLett.88.134502

[2] Gupta, M.R., Banerjee, B., Mandal, L.K., Bhar, R., Pant, H.C., Khan, M. and Srivastava, M.K. (2012) Effect of Viscosity and Surface Tension on the Growth of Rayleigh-Taylor Instability and Richtmyer-Meshkov Instability Induced Two Fluid Interfacial Nonlinear Structure. Indian Journal of Physics, 86, 471-479. http://dx.doi.org/10.1007/s12648-012-0077-3

[3] Gupta, N.K. and Lawande, S.V. (1986) Rayleigh-Taylor Instability in Multi-Structured Spherical Targets. Plasma Physics and Controlled Fusion, 28, 925-941. http://dx.doi.org/10.1088/0741-3335/28/6/008

[4] Hazak, G. (1996) Lagrangian Formalism for the Rayleigh-Taylor Instability. Physical Review Letters, 76, 4167-4170. http://dx.doi.org/10.1103/PhysRevLett.76.4167

[5] Hecht, J., Alon, U. and Shvarts, D. (1994) Potential Flow Models of Rayleigh-Taylor and Richtmyer-Meshkov Bubble Fronts. Physics of Fluids, 6, 4019-4030. http://dx.doi.org/10.1063/1.868391

[6] Piriz, A.R., Lpez Cela, J.J. and Cortzar, O.D. (2005) Rayleigh-Taylor Instability in Elastic Solids. Physical Review E, 72, Article ID: 056313. http://dx.doi.org/10.1103/PhysRevE.72.056313

[7] Ramaprabhu, P. and Dimonte, G. (2005) Single-Mode Dynamics of the Rayleigh-Taylor Instability at Any Density Ratio. Physical Review E, 71, Article ID: 036314. http://dx.doi.org/10.1103/PhysRevE.71.036314

[8] Sohn, S.-I. (2003) Simple Potential-Flow Model of Rayleigh-Taylor and Richtmyer-Meshkov Instabilities for All Density Ratios. Physical Review E, 67, Article ID: 026301.

[9] Velikovich, A.L. and Dimonte, G. (1996) Nonlinear Perturbation Theory of the Incompressible Richtmyer-Meshkov Instability. Physical Review Letters, 76, 3112. http://dx.doi.org/10.1103/PhysRevLett.76.3112

[10] Zhang, Q. (1998) Analytical Solutions of Layzer-Type Approach to Unstable Interfacial Fluid Mixing. Physical Review Letters, 81, 3391-3394. http://dx.doi.org/10.1103/PhysRevLett.81.3391

[11] Banerjee, R., Mandal, L.K., Khan, M. and Gupta, M.R. (2013) Bubble and Spike Growth Rate of Rayleigh Taylor and Richtmeyer Meshkov Instability in Finite Layers. Indian Journal of Physics, 87, 929-937. http://dx.doi.org/10.1007/s12648-013-0300-x

[12] Smalyuk, V.A., Barrios, M., Caggiano, J.A., et al. (2014) Hydrodynamic Instability Growth and Mix Experiments at the National Ignition Facilitya. Physics of Plasmas, 21, Article ID: 056301. http://dx.doi.org/10.1063/1.4872026

[13] Edwards, M.J., et al. (2013) Progress towards Ignition on the National Ignition Facility. Physics of Plasmas, 20, Article ID: 070501.

[14] Mason, R.J. and Tabak, M. (1998) Magnetic Field Generation in High-Intensity-Laser-Matter Interactions. Physical Review Letters, 80, 524-527. http://dx.doi.org/10.1103/PhysRevLett.80.524

[15] Sudan, R.N. (1993) Mechanism for the Generation of 109 G Magnetic Fields in the Interaction of Ultraintense Short Laser Pulse with an Overdense Plasma Target. Physical Review Letters, 70, 3075-3078. http://dx.doi.org/10.1103/PhysRevLett.70.3075

[16] Wilks, S.C., Kruer, W.L., Tabak, M. and Langdon, A.B. (1992) Absorption of Ultra-Intense Laser Pulses. Physical Review Letters, 69, 1383-1386. http://dx.doi.org/10.1103/PhysRevLett.69.1383

[17] Srivastava, M.K., Lawande, S.V., Khan, M., Das, C. and Chakraborty, B. (1992) Axial Magnetic Field Generation by Ponderomotive Force in a Laser-Produced Plasma. Physics of Fluids B, 4, 4086. http://dx.doi.org/10.1063/1.860315

[18] Khan, M., Das, C., Chakraborty, B., et al. (1998) Self-Generated Magnetic Field and Faraday Rotation in a LaserProduced Plasma. Physical Review E, 58, 925-930. http://dx.doi.org/10.1103/PhysRevE.58.925 
[19] Evans, R.G. (1986) The Influence of Self-Generated Magnetic Fields on the Rayleigh-Taylor Instability. Plasma Physics and Controlled Fusion, 28, 1021. http://dx.doi.org/10.1088/0741-3335/28/7/006

[20] Ghezzi, C.R., de Gouveia Dal Pino, E.M. and Horvath, J.E. (2001) Magnetic Field Effects on the Thermonuclear Combustion Front of Chandrasekhar Mass White Dwarfs. The Astrophysical Journal Letters, 548, L193. http://dx.doi.org/10.1086/319091

[21] de Gouveia Dal Pino, E.M. and Benz, W. (1993) Three-Dimensional Simulations of Protostellar Jets. Astrophysical Journal, 410, 686-695. http://dx.doi.org/10.1086/172785

[22] Chandrasekhar, S. (1961) Hydrodynamic and Hydromagnetic Stability. Dover Publications, Inc., New York.

[23] Liberatore, S. and Bouquet, S. (2008) Analytical Modeling of Magnetic Rayleigh-Taylor Instabilities in Compressible Fluids. Physics of Fluids, 20, Article ID: 116101. http://dx.doi.org/10.1063/1.3025832

[24] Samtaney, R. (2003) Suppression of the Richtmyer-Meshkov Instability in the Presence of a Magnetic Field. Physics of Fluids, 15, L53. http://dx.doi.org/10.1063/1.1591188

[25] Jun, B.I., Norman, M.L. and Stone, J.M. (1995) A Numerical Study of Rayleigh-Taylor Instability in Magnetic Fluids. The Astrophysical Journal, 453, 322. http://dx.doi.org/10.1086/176393

[26] Gupta, M.R., Roy, S., Khan, M., et al. (2009) Effect of Compressibility on the Rayleigh-Taylor and RichtmyerMeshkov Instability Induced Nonlinear Structure at Two Fluid Interface. Physics of Plasmas, 16, Article ID: 032303. http://dx.doi.org/10.1063/1.3074789

[27] Gupta, M.R., Mandal, L.K., Roy, S. and Khan, M. (2010) Effect of Magnetic Field on Temporal Development of Rayleigh-Taylor Instability Induced Interfacial Nonlinear Structure. Physics of Plasmas, 17, Article ID: 012306. http://dx.doi.org/10.1063/1.3293120

[28] Layzer, D. (1955) On the Instability of Superposed Fluids in a Gravitational Field. The Astrophysical Journal, 122, 1. http://dx.doi.org/10.1086/146048

[29] Drake, R.P. (2006) High Energy-Density Physics. Springer, New York.

[30] Casali, R., Castro, L.B. and Menezes, D.P. (2014) Hadronic and Hybrid Stars Subject to Density-Dependent Magnetic Fields. Physical Review C, 89, Article ID: 015805. http://dx.doi.org/10.1103/PhysRevC.89.015805

[31] Lamb, H. (1932) Hydrodynamics. Cambridge University Press, Cambridge.

[32] Bernstein, I.B. and Book, D.L. (1983) Effect of Compressibility on the Rayleigh-Taylor Instability. Physics of Fluids, 26, 453. http://dx.doi.org/10.1063/1.864158 